\title{
An unusual cause of transient ischemic attack
}

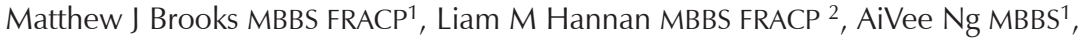 \\ Louis Irving MBBS FRACGP FRACP ${ }^{3}$, Anu Aggarwal MBBS FRACP PhD ${ }^{1}$
}

\begin{abstract}
Tn April 2011, a 57-year-old woman with a long smoking history presented with transient symptoms of left-hand incoordination and dysarthria. At the time of arrival to hospital, there had been complete resolution of neurological signs and symptoms. Computed tomography and subsequent magnetic resonance imaging of the brain demonstrated no evidence of cerebral infarction and no other intracranial pathology. A routine chest radiograph revealed right middle lobe collapse. Computed tomography of the chest demonstrated a large proximal obstructing mass within the right middle lobe in addition to multiple bilateral pulmonary nodules suggestive of an advanced primary bronchogenic carcinoma with pulmonary metastases. There appeared to be tumour extension into the lateral wall of the left atrium (Figures $1 \mathrm{~A}$ and 1B). Standard bronchoscopy demonstrated an endobronchial tumour at the origin of the right middle lobe bronchus, and endobronchial biopsy and brush cytology confirmed an adenocarcinoma that was TTF-1 and cytokeratin positive, consistent with a primary lung malignancy. A transesophageal echocardiogram demonstrated tumour infiltration of the right lower pulmonary vein with extension into the left atrium (Figure 2). There was no evidence of vegetations on the aortic or mitral valves, effectively excluding the possibility of marantic endocarditis. No other alternative sources of emboli were identified to explain the transient ischemic attack. With the exception of atrial myxoma, embolic phenomena due to intracardiac tumours are uncommon. Previous case reports of intracardiac extension of primary lung tumours have generally described extension via the pulmonary vein, as was apparent in the present case (1). The patient was referred for palliative radiotherapy for the primary lesion in addition to systemic chemotherapy.
\end{abstract}
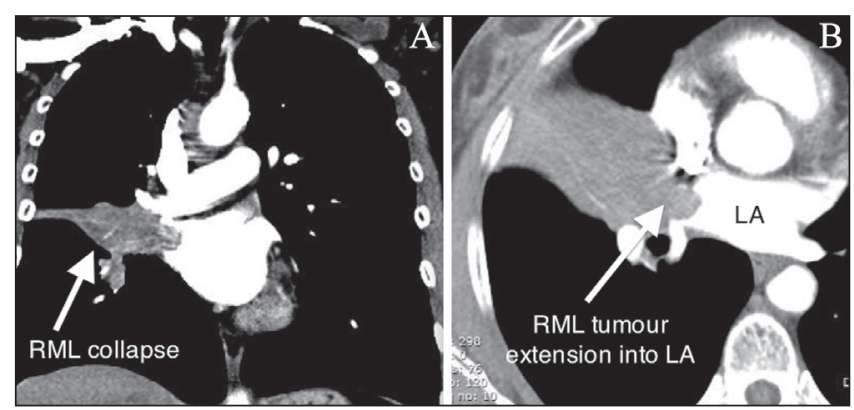

Figure 1) Chest computed tomography. A Coronal image demonstrating right middle lobe (RML) collapse from obstructing tumour. B Axial image demonstrating tumour extension into the right lower pulmonary vein and left atrium (LA)

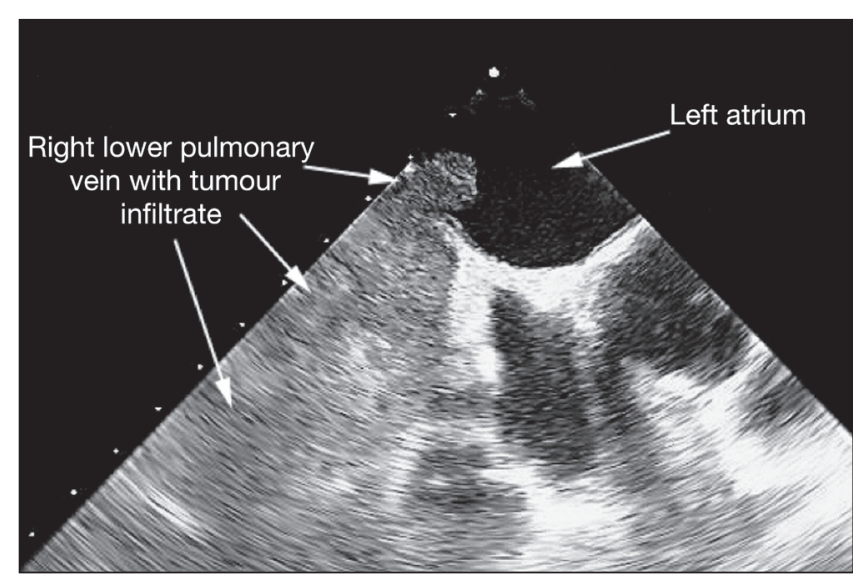

Figure 2) Transesophageal echocardiogram image demonstrating extensive tumour infiltration of the right lower pulmonary vein and extension into the left atrium

\section{LEARNING POINTS}

1. While the occurrence of cerebral infarction or transient ischemic attack in association with lung cancer is most likely to be due to one of the common causes of stroke (such as carotid atheroma or atrial fibrillation), rarer causes, such as marantic endocarditis or direct extension of tumour into vascular structures, should also be considered.

2. Transesophageal echocardiography is able to reliably identify the presence of vegetations on heart valves and may also provide additional information on pulmonary lesions in close proximity to cardiac structures $(2,3)$.

\section{REFERENCES}

1. Kodama K, Doi O, Tatsuta M. Unusual extension of lung cancer into the left atrium via the pulmonary vein. Int Surg 1990;75:22-6.

2. Wang KY, Lin CY, Kuo-Tai J, Yuan L, Chang HJ. Use of transesophageal echocardiography for evaluation of resectability of lung cancer. Acta Anaesthesiologica Sinica 1994;32:255-60.

3. Caterino U, Dialetto G, Covino FE, Mazzarella G, Grella E, Massimo M. The usefulness of transesophageal echocardiography in the staging of locally advanced lung cancer. Monaldi Arch Chest Dis 2007;67:39-42.

The 'Images in Respiratory Medicine' section of the Canadian Respiratory Journal aims to highlight the importance of visual interpretation, whether physiological, radiological, bronchoscopic, surgical/thorascopic or histological, in the diagnosis of chest diseases. Submissions should exemplify a classic, particularly dramatic or intriguing presentation of a disease while offering an important educational message to the reader (insightful diagnostic pearls or differential diagnosis, etc). This section is not intended to be a vehicle for publication of case reports (see the Clinical-Pathologic Conferences for case-based leaning series).

${ }^{1}$ Department of Cardiology, Royal Melbourne Hospital; ${ }^{2}$ Institute for Breathing and Sleep, Heidelberg; ${ }^{3}$ Department of Respiratory and Sleep Medicine, Royal Melbourne Hospital, Melbourne, Victoria, Australia

Correspondence: Dr Liam Hannan, Institute for Breathing and Sleep, 145 Studley Road, Heidelberg, Melbourne, Victoria 3084, Australia.

Telephone 778-8361863, e-mail liam.hannan@austin.org.au 


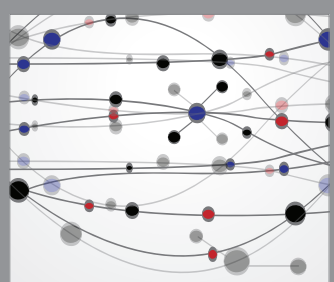

The Scientific World Journal
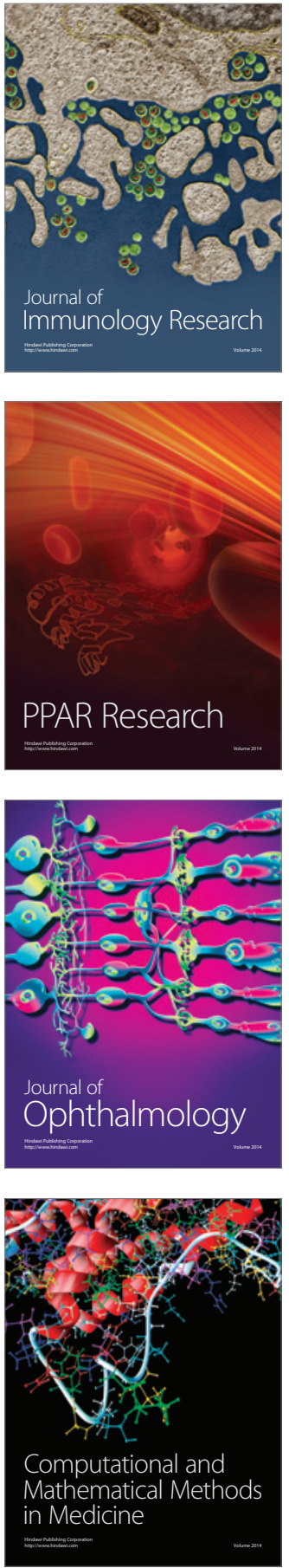

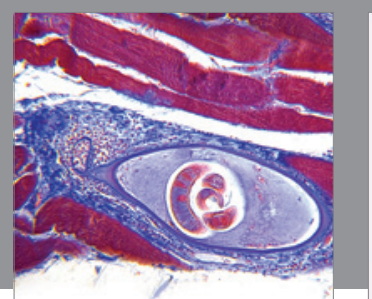

Gastroenterology Research and Practice

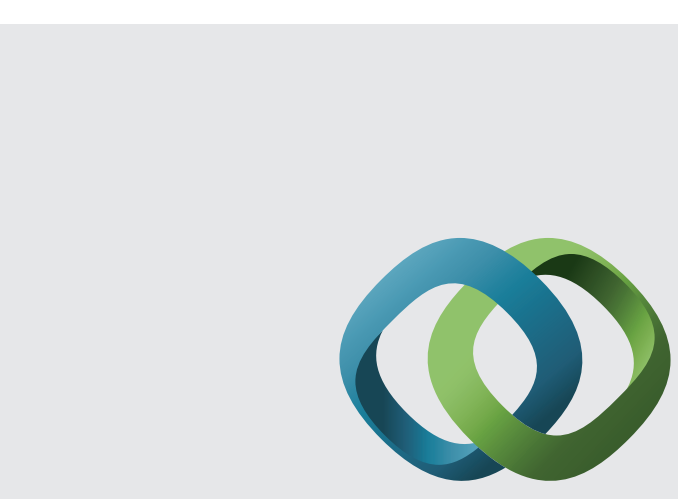

\section{Hindawi}

Submit your manuscripts at

http://www.hindawi.com
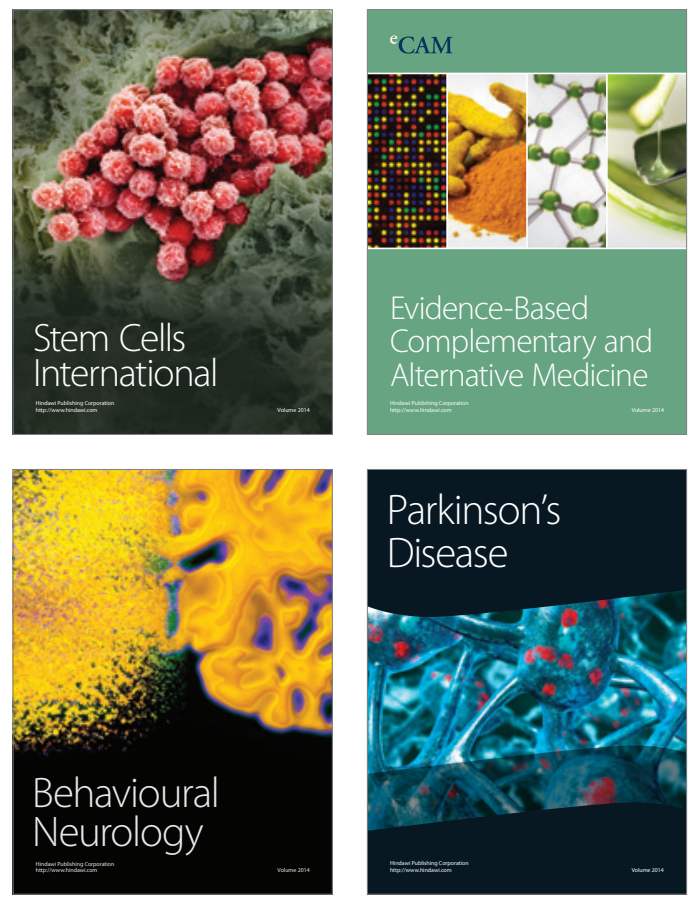
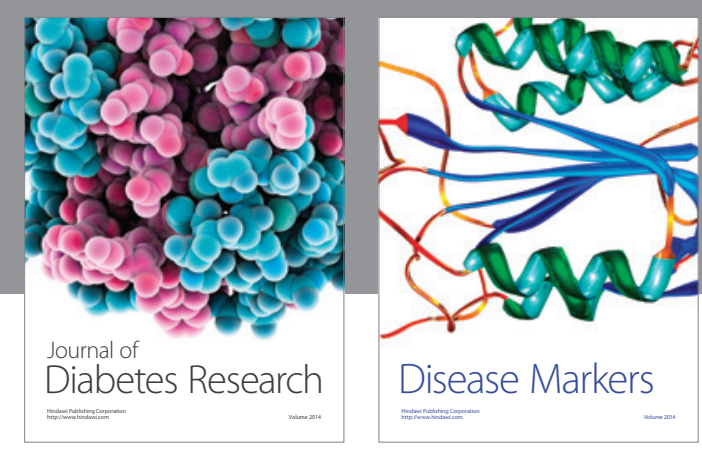

Disease Markers
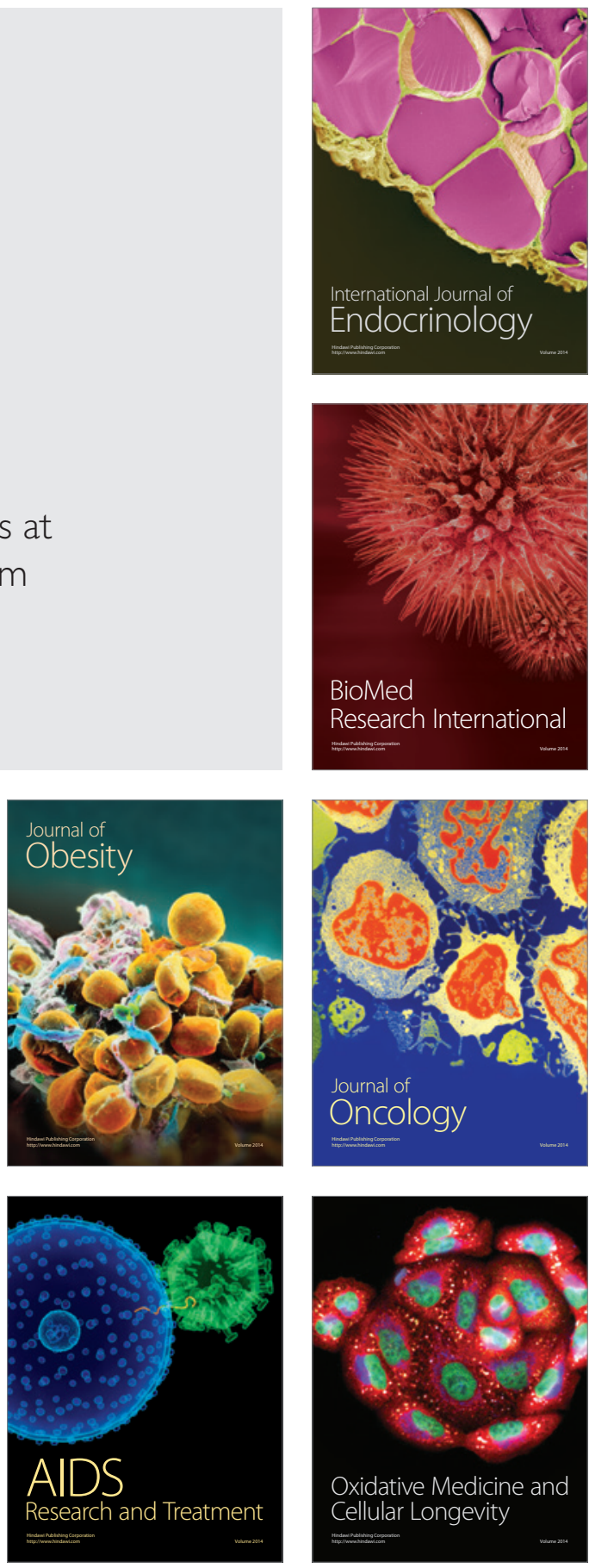